\title{
Comprehensive evaluation of NT5E/CD73 expression and its prognostic significance in distinct types of cancers
}

Tao Jiang ${ }^{1 \dagger}$, Xiaofeng Xu ${ }^{2,3+}$, Meng Qiao ${ }^{1}$, Xuefei $\mathrm{Li}^{4}$, Chao Zhao ${ }^{4}$, Fei Zhou' ${ }^{1}$ Guanghui Gao ${ }^{1}$, Fengying $\mathrm{Wu}^{1}$, Xiaoxia Chen ${ }^{1}$, Chunxia Su', Shengxiang Ren ${ }^{1}$, Changyun Zhai ${ }^{5^{*}}$ and Caicun Zhou ${ }^{1 *}$

\begin{abstract}
Background: CD73 is one of the critical component in the formation of immunosuppressive microenvironment in cancers. We aimed to provide an overview of the current status of CD73 expression and its relationship with clinicopathlogical features and prognosis in different cancers.

Methods: PubMed, Web of Science, EMBASE and Cochrane library were searched to identify the relevant studies. CD73 expression level in distinct cancers and its relationship with clinicopathlogical characteristics and prognosis were investigated using online database. Meta-analyses were conducted using RevMan v5.0 and STATA v12.0.

Results: Fourteen publications with 2951 cases were included. The incidence of high CD73 expression was 0.50 (95\% Cl: 0.36-0.63). Data from Oncomine validated that median CD73 expression level in tumor tissues was markedly higher than that in normal tissues in most kinds of cancers except cecum adenocarcinoma and ovarian cancer $(P<0.05)$. High CD73 expression was significantly correlated with shorter overall survival (OS) in various cancers (high risk $[H R]=1.48 ; P<0.05$ ). Subgroup analysis using online database demonstrated that high CD73 expression was significantly correlated with poor $O S$ in breast $(H R=1.23 ; P<0.05)$ and ovarian cancer $(H R=1.14 ; P$ $<0.05)$, but favorable OS in lung ( $H R=0.80 ; P<0.05)$ and gastric cancer $(H R=0.71 ; P<0.05)$. High $C D 73$ expression was dramatically associated with lymph node metastases $(\mathrm{OR}=2.61 ; P=0.05)$.
\end{abstract}

Conclusion: High CD73 expression was significantly associated with lymph node metastases and a promising prognostic factor in different types of cancers.

Keywords: CD73, Cancer, Immunotherapy, Prognosis, Characterization, Meta-analysis

\section{Background}

CD73, also designated ecto-5' -nucleotidase (NT5E), is one kind of ecto-nucleotidase that plays a critical role in the catabolism of extracellular ATP to adenosine and the maintenance of immune homeostasis [1, 2]. CD73 is the rate-limiting enzyme in the ATP to adenosine degradation pathway. It can dephosphorylate adenosine

\footnotetext{
*Correspondence: zaichangyun@163.com; caicunzhou_dr@163.com ${ }^{\dagger}$ Equal contributors

${ }^{5}$ Department of Medical Oncology, Yancheng TCM Hospital Affiliated to Nanjing University of Chinese Medicine, Yancheng 224001, People's Republic of China

'Department of Medical Oncology, Shanghai Pulmonary Hospital, Thoracic Cancer Institute, Tongji University School of Medicine, No. 507, Zheng Min Road, Shanghai 200433, People's Republic of China

Full list of author information is available at the end of the article
}

monophosphate (AMP) to form adenosine and activate specific G-protein coupled receptor (GPCR) to increase intracellular cAMP level, thus promoting cancer cell aggressiveness, metastasis and angiogenesis [3-7]. Previous studies unraveled that extracellular adenosine concentration was elevated in the tumor microenvironment [8]. Recently, CD73-adensine was found to be a significant pathway involved in the formation of immunosuppressive microenvironment in distinct tumors [3].

CD73-derived adenosine mainly mediates immunosuppression via activation of A2A receptor on immune cells, especially natural killer (NK) cells and CD8+ T cells. Recent studies revealed that CD73 plays a pivotal role in tumor escape from immune surveillance. The mechanism can be summarized into three aspects: (i) inhibition 
of clonal expansion, activation and homing to tumor specific $\mathrm{T}$ cells; (ii) to increase a substantial component of the suppressive capabilities of regulatory $\mathrm{T}$ cells (Tregs) and Th17 cells; (iii) to accelerate the conversion of anti-tumor type 1 macrophages into pro-tumor type 2 macrophages [9]. Targeting CD73 results in favorable antitumor effects in preclinical studies and combination of CD73 blockade with other immune checkpoint inhibitors, such as anti-cytotoxic T-lymphocyte antigen (CTLA)-4 antibody or anti-programmed cell death protein (PD)-1/PD-1 ligand (PDL1) antibody, is particularly promising [9]. Increasing evidence suggested that CD73 highly expressed in a wide range of cancer types, including breast cancer, colorectal cancer, glioblastoma, melanoma, prostate cancer, ovarian cancer, and non-small-cell lung cancer (NSCLC). High CD73 expression was often associated with poor prognosis in different cancers. However, several studies demonstrated that high CD73 expression was not correlated with the prognosis of patients with breast cancer [10]. Even some studies indicated that high CD73 expression was associated with favorable prognosis in patients with gastric cancer or rectal adenocarcinoma $[11,12]$.

To date, there is no study to comprehensively investigate the correlation between high CD73 expression and prognosis in cancer patients. There is also no study to dissect the CD73 expression level in different cancers and the relationship between high CD73 expression and clinicopathlogical characteristics. Herein, we conducted this study with published data and online database to clarify the influence of high CD73 expression and its impact on the outcomes of different cancers, as well as its relationship with clinicopathlogical features. Furthermore, we performed subgroup analysis on the association of high CD73 expression with prognosis in breast, lung, gastric and ovarian cancer by using the published data on KM plotter (http://www.kmplot.com). We aimed to provide an overview of the current status of high CD73 expression in tumor prognosis and future immunotherapy.

\section{Methods}

\section{Online search}

We carried out a publication search through PubMed/ Medline, EMBASE, Google Scholar, Cochrane Library and Web of Science until January 31, 2017 (records in English or Chinese). The following keywords was utilized: ("CD73" OR "NT5E" OR "ecto-5'-nucleotidase") and ("cancer" OR "tumor" OR "carcinoma" OR "neoplasm"). We firstly reviewed the titles and abstracts to determine publications, which investigated the relationship of CD73 expression with overall survival (OS), recurrence free survival (RFS) and clinicopathological characteristics. Reference in each articles were hand- searched. This analysis was conducted in line with Preferred Reporting Items for Systematic Reviews and Meta-Analyses: the PRISMA Statement [13].

\section{Publication selection}

Publications met the following criteria were eligible: (1) investigated high CD73 expression in kinds of human solid tumors; (2) CD73 expression was determined on tumor specimens, instead of the peripheral blood or cell lines or any other types of tissue; (3) reported data could analyze the rate of high CD73 expression and/or high risk (HR) on clinical outcomes. Studies were ineligible if they were: (1) comment, reviews, case-only studies, editorial, or familial studies; (2) insufficient data for analysis of rate and/or HR with 95\% confidence intervals (CIs); and (3) repeat of previous publications or replicated samples. The study eligibility was independently evaluated by two reviewers. Disagreements were resolved after discussion with third reviewer.

\section{Data extraction and quality assessment}

We extracted the following information from the included studies: name of first author, publication year, tumor types, study population, high CD73 expression test techniques, cut-off value, and rate of high CD73 expression with $95 \%$ CIs, HR for DFS, RFS, and/or OS with related 95\% CIs. If the HRs and CIs were not reported, the total death events and the numbers of study population in each group were extracted to indirectly analyze HRs and CIs. To avoid the selection bias, we did not extracted data from the reported Kaplan-Meier curves. When univariate and multivariate analysis were simultaneously reported, the results of multivariate analysis were selected. Two reviewers independently extracted the data by using a predefined Excel form. Disagreements were solved by consensus. As we previously mentioned [14], two reviewers assessed the study quality independently by using the listed factors. Studies lacking any of these criteria would also be excluded.

\section{Online database cross-validation}

To determine the expression level of CD73 in a broader set of cancers and matched normal tissues, we queried the Oncomine, a web-based microarray database (http:// www.oncomine.org), to analyze the gene expression level of CD73 in more than 20 types of cancers with distinct histology. We then examined the association of high CD73 expression with prognosis in breast, lung, gastric and ovarian cancer by using the published data on http://www.kmplot.com.

\section{Statistical analysis}

The incidence of high CD73 expression were combined. Respective 95\% CIs were determined per estimate and 
presented in forest plots. For time-to-event data, the HRs with related 95\% CIs were directly extracted from the eligible publications or calculated using previous methods proposed by Tierney et al. [15]. Cochran's Q test and $\mathrm{I}^{2}$ statistic were used to determine the heterogeneity of different studies. Low-level heterogeneity was defined as $P>0.1$ for the $X^{2}$ test and $\mathrm{I}^{2}<25 \%$. If the heterogeneity was non-significant, a pooled effect was calculated with a fixed-effects model. A random-effects model was used when the heterogeneity was statistically significant. Publication bias was assessed by using funnel plots, Begg's and Egger's tests. Statistical analysis was conducted by Review Manager 5.0 software and STATA v12.0 (Stata Corporation, TX). All data were analyzed using the Statistical Package for Social Sciences (SPSS) software (version 20.0 for Windows). $P$ values were twosided and considered significant if less than 0.05 except for the Q-test.

\section{Results}

\section{Characteristics of included studies}

The result of studies inclusion was listed in Fig. 1. Briefly, a total of 359 potentially relevant publications were found, and 14 studies were finally included in this study after screening [10-12, 16-26]. Most of the excluded abstracts were reviews, comment or studies with incomplete data. In the current analysis, 2951 cases from 14 studies were applied to explore high CD73 expression in 12 types of human cancers. Three studies were in CRC, 2 studies were in ovarian cancer and other 9 studies were about breast, digestive, gynecological, urinary and lung cancer. The main characteristics of the included studies were shown in Table 1. In addition, prognostic data were obtained from all of included studies on OS and 4 of 14 studies on RFS.

\section{Test method of high CD73 expression}

Immunohistochemistry (IHC), immunofluorescence (IF) and microarray analysis (validated with another method) were used to test CD73 expression. IHC was the most commonly used method (12 of 14). Of note, the criteria for high CD73 expression were distinct among different studies using IHC. For example, in some studies, the percentage of positive-staining tumor cells larger than median expression level were considered to be high CD73 expression. In other studies, staining intensity > $10 \%$ of positive-staining tumor cells was taken as high CD73 expression. Semi-quantitative 3 -scale scoring system and 4-value grade were commonly used criterion, which were obtained for each case by multiplying the percentage and intensity score. The definition of positive expression of CD73 were summarized in Table 1 . Nevertheless, in these studies used this scoring system, the cutoff points were distinct among different studies.

\section{Prevalence of high CD73 expression}

The incidence of high CD73 expression in these studies ranged from $10.30 \%$ to $74.30 \%$, partly reflecting the heterogeneity in the criteria for high expression. In the meta-analysis of 14 studies, the incidence of high CD73 expression was 0.50 (95\% CI: $0.36-0.63)$ and large heterogeneity existed $\left(\mathrm{I}^{2}=98.0 \% ; P<0.05\right.$; Fig. 2$)$. Subgroup analysis was stratified by test methods (IHC) and evaluation criteria, but the heterogeneity could not be reduced.

The expression level of CD73 in different cancers were explored by using the data from Oncomine. As shown in Supplemental Material, median CD73 expression level in tumor tissues was significantly higher than that in normal tissues in most kinds of cancers including bladder, brain, invasive lobular breast, esophageal, gastric,

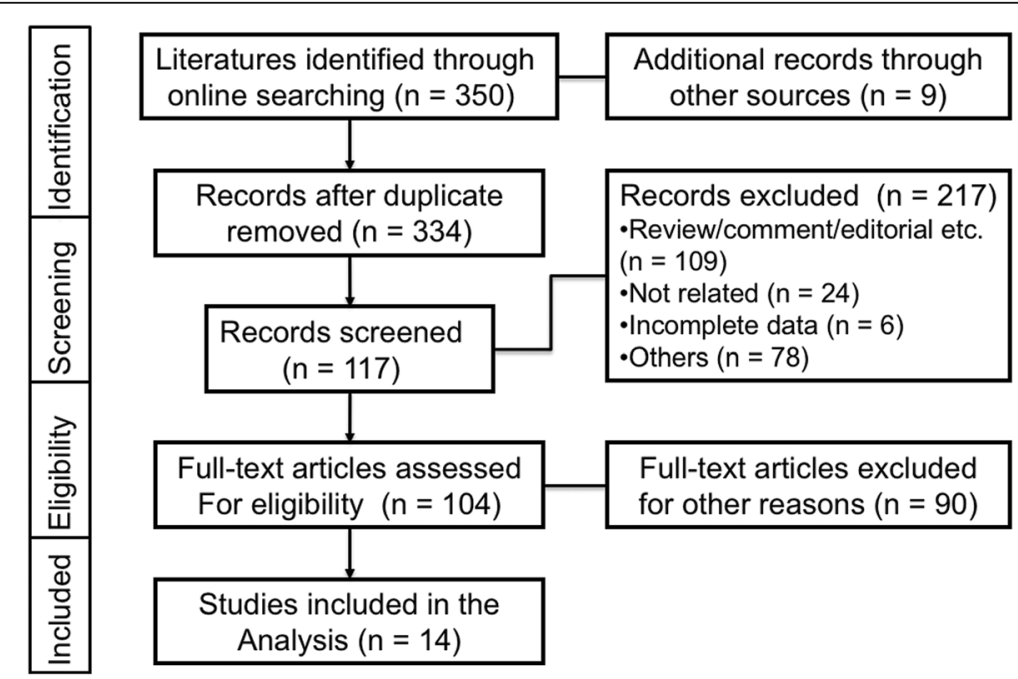

Fig. 1 Flowchart of the study inclusion 
Table 1 Baseline Characteristics of included studies

\begin{tabular}{|c|c|c|c|c|c|c|c|}
\hline Author & Tumor type & Year & No. of cases & CD73+ No. & Positive rate & Test methods & Definition of high expression \\
\hline Hoon et al. & Ovarian cancer & 2012 & 167 & 117 & $70.10 \%$ & $\mathrm{IHC}$ & $\begin{array}{l}\text { using a 4-value grade } \\
(>0 \text { score })^{*}\end{array}$ \\
\hline Anna et al. & Breast cancer & 2012 & 136 & 101 & $74.30 \%$ & $\mathrm{IHC}$ & $>$ median expression levels \\
\hline Wu et al. & Colorectal Cancer & 2012 & 223 & 100 & $44.80 \%$ & $\mathrm{IHC}$ & $\begin{array}{l}\text { using } X \text {-tile program to } \\
\text { determine the cutoff value }\end{array}$ \\
\hline Wu et al. & Colorectal Cancer & 2012 & 135 & 68 & $50.40 \%$ & $\mathrm{IHC}$ & $\begin{array}{l}\text { using } X \text {-tile program to } \\
\text { determine the cutoff value }\end{array}$ \\
\hline Lu et al. & Gastric cancer & 2013 & 68 & 31 & $45.60 \%$ & $\mathrm{IHC}$ & semi-quantitative method $>5^{* *}$ \\
\hline Xiong et al. & Gallbladder cancer & 2014 & 108 & 59 & $54.60 \%$ & $\mathrm{IHC}$ & $\begin{array}{l}\text { the percent of positively } \\
\text { stained cells was }>10 \%\end{array}$ \\
\hline Martin et al. & Ovarian cancer & 2015 & 208 & 104 & $50.00 \%$ & $\mathrm{IHC}$ & $\begin{array}{l}\text { highest 20\% CD73 expression } \\
\text { was used as a cutoff }\end{array}$ \\
\hline Marian et al. & Bladder Cancer & 2015 & 174 & 46 & $26.40 \%$ & $\mathrm{IHC}$ & $\begin{array}{l}\text { semi-quantitative } 3 \text {-scale } \\
\text { scoring system: }=2^{* * *}\end{array}$ \\
\hline Yu et al. & Renal cell carcinoma & 2015 & 159 & 75 & $47.20 \%$ & $\mathrm{IHC}$ & $\begin{array}{l}\text { using a 4-value intensity score } \\
\text { ( }>2 \text { score) }\end{array}$ \\
\hline Zhang et al. & Rectal adenocarcinoma & 2015 & 90 & 47 & $52.20 \%$ & $\mathrm{IHC}$ & > median expression levels \\
\hline Bruno et al. & Prostate Cancer & 2015 & 285 & NA & NA & IF & $\begin{array}{l}\text { > median mean fluorescence } \\
\text { intensity }\end{array}$ \\
\hline Ren et al. & HNSCC & 2016 & 162 & 100 & $61.70 \%$ & $\mathrm{IHC}$ & $>$ median expression levels \\
\hline Ren et al. & Oral squamous cell carcinoma & 2016 & 113 & 66 & $58.40 \%$ & $\mathrm{IHC}$ & $>10 \%$ positively stained cells \\
\hline Zhang et al. & Colorectal Cancer & 2016 & 566 & 283 & $50.00 \%$ & MA & > median expression levels \\
\hline Yusuke et al. & Non-small-cell lung cancer & 2017 & 642 & 66 & $10.30 \%$ & $\mathrm{IHC}$ & $\begin{array}{l}\text { H-scores that met or exceeded } \\
\text { the individual cutoffs }\end{array}$ \\
\hline
\end{tabular}

*4-value grade: CD73 expression levels were graded on a scale of 0 to 3 based on cytoplasmic and membrane staining intensity and the proportion of positive tumor cells by an expert pathologist who was blinded to the patient's clinical records. The staining was graded as 0 if no cancer cells were reactive, 1 if staining was weakly positive in $<1 / 3$ of cancer cells, 2 if staining was weakly positive in $>2 / 3$ of cancer cells, or strongly positive in $>1 / 3$ of cancer cells, and 3 if staining was weakly positive in most cancer cells, or strongly positive in $>2 / 3$ of cancer cells. Immunohistochemical staining for CD73 in ovarian cancer tissue was classified as negative (grade 0 ) or positive (grade 1 to 3 ).

**Semi-quantitative method: The percentage of positive cells was scored 0 for staining of $<1 \%, 1$ for staining of $2 \%-25 \%, 2$ for staining of $26 \%$ - $50 \%, 3$ for staining of $51 \%-75 \%$, and 4 for staining $>75 \%$ of the cells examined. Staining intensity was calculated, no coloring, slightly yellow, brown yellow and tan stains were marked as $0,1,2$ and 3 . Finally, we calculated the product of staining intensity and positive cell percentage: $\leq 5$ was de ned as negative and $\geq 6$ as positive. *** Semi-quantitative 3 -scale scoring system, score 0: no staining; score 1+: weak staining; score 2+: strong staining.

$\mathrm{H}$-scores were calculated by multiplying the intensity score ( 0 , absent; 1 , weak; 2 , moderate; 3 , strong) by the percentage of stained cells ( $0-100 \%)$ to yield a value of $0-300$.

No., number; IHC, Immunohistochemistry; IF, immunofluoresence; MA, microarray analysis; HNSCC, Head and neck squamous cell carcinoma; NA, not applicable.

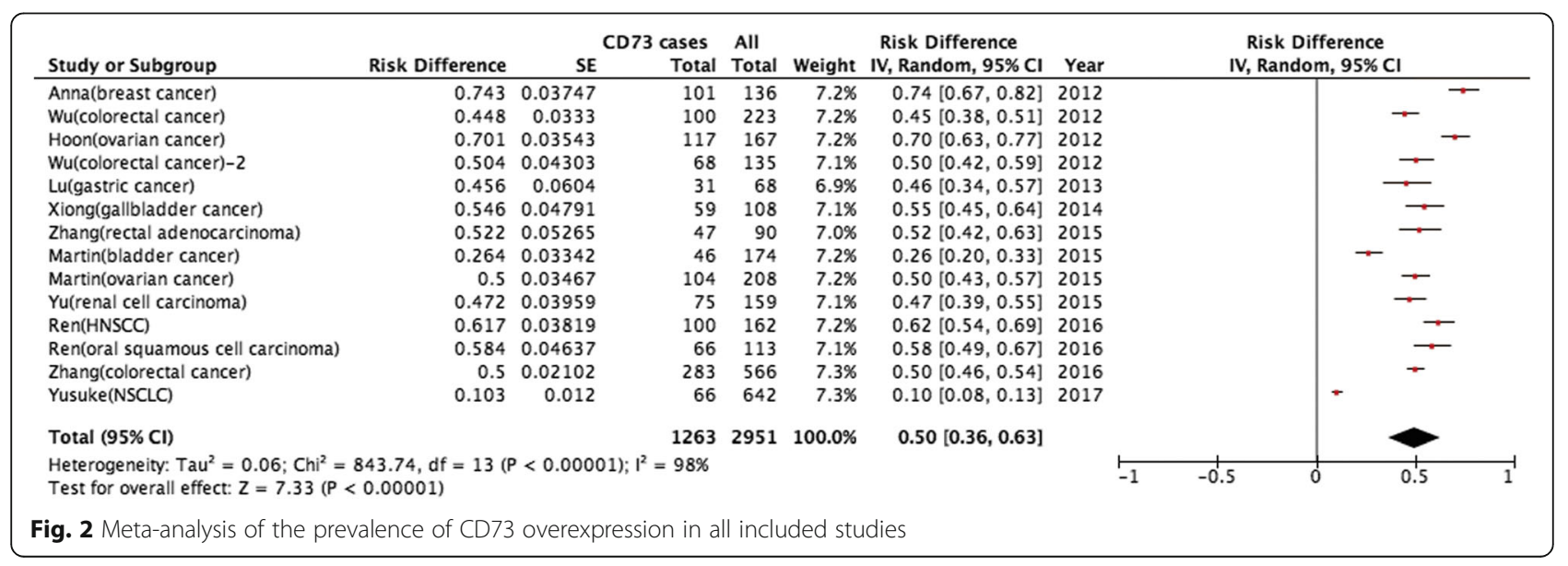


pancreatic cancer, rectal mucinous, renal cell, lung large cell, oral cavity squamous cell carcinoma, melanoma, and lung adenocarcinoma $(P<0.05)$ (Additional file 1: Figures S1, S2, S3, S5, S6, S7, S8, S11, S12, S13, S15, S17). However, several types of tumors (cervical, liver, colorectal, prostate invasive ductal breast, small cell lung cancer and lung squamous cell carcinoma) showed similar CD73 expression level compared to the level in matched normal tissues $(P>$ 0.05) (Additional file 1: Figures S3, S4, S5, S9, S10, S11, S12, S14, S18, S19). Notably, CD73 expression in cecum adenocarcinoma or ovarian cancer was markedly lower than that in matched normal tissue $(P<0.05)$ (Additional file 1: Figures S5, S16). CD73 expression level in different histological types of one cancer was heterogeneous. For example, invasive lobular breast cancer has the higher CD73 expression level while invasive ductal breast cancer has the lower CD73 expression level (Additional file 1: Figure S3). In lung cancer, histology of large cell carcinoma has the significantly higher CD73 expression level but histology of small cell lung cancer and squamous cell carcinoma has the markedly lower expression level than that in matched normal tissue (Additional file 1: Figure S11).

\section{Relationship between high CD73 expression and prognosis}

Pooled analysis was used to assess high CD73 expression overall effect for the studies containing prognostic data. The results showed that high CD73 expression was significantly correlated with poorer OS in various cancers [HR 1.48 (95\% CI: 1.04-2.10); $P=0.030$ ] but large heterogeneity existed $\left(\mathrm{I}^{2}=78.0 \% ; P<0.05\right.$; Fig. $\left.3 \mathrm{a}\right)$. In the four studies that reported RFS, the pooled result indicated that high CD73 expression was not associated with RFS [HR: 1.42 (95\% CI: $0.82-2.45$ ); $P=0.210$; Fig. 3b]. The results also showed high heterogeneity $\left(\mathrm{I}^{2}=77.0 \% ; P<0.05\right)$.

In addition, we carried out the subgroup analysis of association between high CD73 expression and prognosis in breast, lung, gastric and ovarian cancer by using online database. The representative figures of high CD73 expression and negative CD73 expression in breast, lung and gastric and ovarian cancer were obtained from the Human Protein Atlas (proteinatlas.org) with the approval and listed in Fig. 4a. Consistent with the metaanalysis, the results from database showed that high CD73 expression was significantly correlated with poor OS in breast [HR: 1.23 (95\% CI: $1.11-1.38$ ); $P<$ 0.05; Fig. 4b] and ovarian cancer [HR: 1.14 (95\% CI: 1.00-1.29); $P<0.05$; Fig. 4e]. However, high CD73 expression was correlated with favorable OS in lung [HR: 0.80 (95\% CI: 0.71-0.91); $P<0.05$; Fig. 4c] and gastric cancer [HR: 0.71 (95\% CI: 0.60-0.84); $P<0.05$; Fig. 4d].

\section{Relationship between high CD73 expression and clinicopathological parameters}

To investigate the relationship between high CD73 expression and clinicopathological features, meta-

\begin{tabular}{|c|c|c|c|c|c|c|c|}
\hline $\begin{array}{l}\text { a } \\
\text { Study or Subgroup }\end{array}$ & log[Hazard Ratio] & SE & Weight & $\begin{array}{l}\text { Hazard Ratio } \\
\text { IV, Random, } 95 \% \mathrm{CI}\end{array}$ & Year & \multicolumn{2}{|c|}{$\begin{array}{c}\text { Hazard Ratio } \\
\text { IV, Random, } 95 \% \mathrm{CI}\end{array}$} \\
\hline Hoon(ovarian cancer) & 0.0296 & 0.4113 & $6.9 \%$ & $1.03[0.46,2.31]$ & 2012 & & \\
\hline Anna(breast cancer) & -1.2379 & 0.6571 & $4.4 \%$ & $0.29[0.08,1.05]$ & 2012 & & \\
\hline Wu(colorectal cancer) & 1.0919 & 0.3605 & $7.6 \%$ & $2.98[1.47,6.04]$ & 2012 & & \\
\hline Wu(colorectal cancer)-2 & 1.1184 & 0.3706 & $7.5 \%$ & $3.06[1.48,6.33]$ & 2012 & & \\
\hline Lu(gastric cancer) & -0.9416 & 0.4237 & $6.8 \%$ & $0.39[0.17,0.89]$ & 2013 & & \\
\hline Xiong(gallbladder cancer) & 0.9361 & 0.3975 & $7.1 \%$ & $2.55[1.17,5.56]$ & 2014 & & \\
\hline Bruno(prostate cancer) & 1.0116 & 0.3161 & $8.2 \%$ & $2.75[1.48,5.11]$ & 2015 & & \\
\hline Zhang(rectal adenocarcinoma) & -0.7133 & 0.3433 & $7.8 \%$ & $0.49[0.25,0.96]$ & 2015 & & \\
\hline Yu(renal cell carcinoma) & 1.3838 & 0.6621 & $4.4 \%$ & $3.99[1.09,14.61]$ & 2015 & & \\
\hline Martin(bladder cancer) & -1.1394 & 0.7754 & $3.6 \%$ & $0.32[0.07,1.46]$ & 2015 & & \\
\hline Martin(ovarian cancer) & 0.1989 & 0.0717 & $10.7 \%$ & $1.22[1.06,1.40]$ & 2015 & & $\rightarrow$ \\
\hline Ren(HNSCC) & 0.8629 & 0.3025 & $8.3 \%$ & $2.37[1.31,4.29]$ & 2016 & & \\
\hline Ren(oral squamous cell carcinoma) & 0.8587 & 0.3712 & $7.4 \%$ & $2.36[1.14,4.89]$ & 2016 & & \\
\hline Yusuke(NSCLC) & 0.7793 & 0.2333 & $9.2 \%$ & $2.18[1.38,3.44]$ & 2017 & & \\
\hline Total $(95 \% \mathrm{CI})$ & & & $100.0 \%$ & $1.48[1.04,2.10]$ & & & \\
\hline $\begin{array}{l}\text { Heterogeneity: } \mathrm{Tau}^{2}=0.30 ; \mathrm{Ch}^{2}= \\
\text { Test for overall effect: } \mathrm{Z}=2.18(\mathrm{P}=\end{array}$ & $\begin{array}{l}58.59, \mathrm{df}=13(\mathrm{P}<\mathrm{C} \\
0.03)\end{array}$ & $0.00001)$ & $; 1^{2}=78 \%$ & & & $\begin{array}{ccc}1 & 1 & 1 \\
0.1 & 0.2 & 0.5 \\
& \text { Favours [high } \mathrm{CD} 73 \text { ] }\end{array}$ & \begin{tabular}{|ccc}
1 & 1 & 1 \\
1 & 5 & 10 \\
Favours [low & CD 73$]$ &
\end{tabular} \\
\hline $\begin{array}{l}\text { D } \\
\text { Study or Subgroup }\end{array}$ & log[Hazard Ratio] & SE & Weight & $\begin{array}{l}\text { Hazard Ratio } \\
\text { IV, Random, 95\% CI }\end{array}$ & Year & $\begin{array}{l}\text { Hazard } \\
\text { IV, Randor }\end{array}$ & $\begin{array}{l}\text { d Ratio } \\
\mathrm{m}, 95 \% \mathrm{Cl}\end{array}$ \\
\hline Anna(breast cancer) & -1.0788 & 0.4905 & $16.9 \%$ & $0.34[0.13,0.89]$ & 2012 & $\longrightarrow$ & \\
\hline Martin(ovarian cancer) & 0.3914 & 0.1738 & $31.0 \%$ & $1.48[1.05,2.08]$ & 2015 & & -- \\
\hline Ren(oral squamous cell carcinoma) & 0.8825 & 0.3737 & $21.6 \%$ & $2.42[1.16,5.03]$ & 2016 & & \\
\hline Yusuke(NSCLC) & 0.7178 & 0.1873 & $30.4 \%$ & $2.05[1.42,2.96]$ & 2017 & & $\rightarrow-$ \\
\hline Total $(95 \% \mathrm{Cl})$ & & & $100.0 \%$ & $1.42[0.82,2.45]$ & & & \\
\hline $\begin{array}{l}\text { Heterogeneity: } \mathrm{Tau}^{2}=0.22 ; \mathrm{Ch}^{2}= \\
\text { Test for overall effect: } \mathrm{Z}=1.25(\mathrm{P}=\end{array}$ & $\begin{array}{l}13.13, \mathrm{df}=3(P=0 \\
0.21)\end{array}$ & $.004) ; 1^{2}=$ & $=77 \%$ & & & $\begin{array}{|cc|}0.01 & 0.1 \\
& \text { Favours [high CD73] }\end{array}$ & $\begin{array}{|cc|}10 & 100 \\
\text { Favours [low CD73] } & \end{array}$ \\
\hline
\end{tabular}




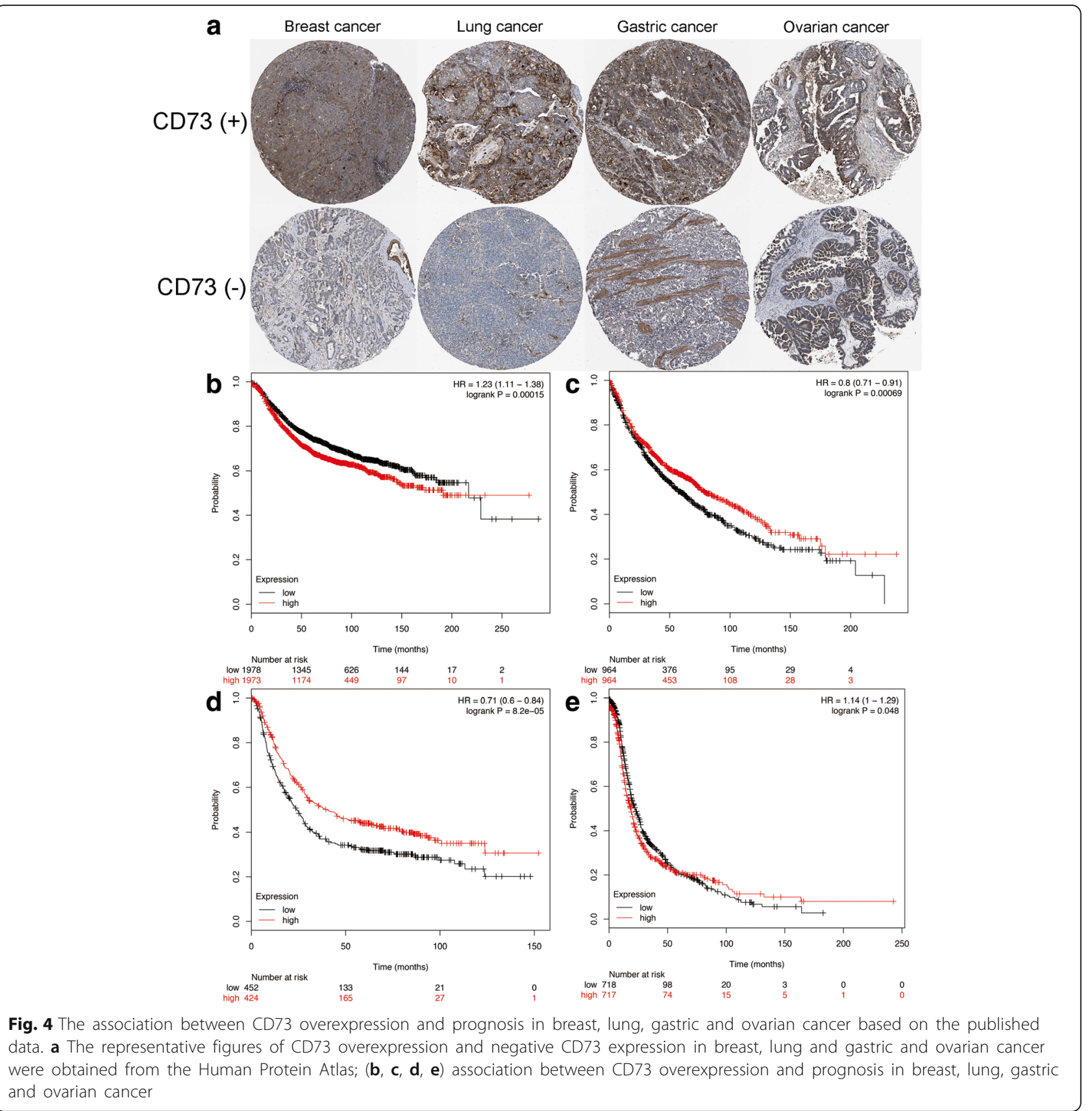

analyses were performed according to the different characteristics. As the results suggested, high CD73 expression was dramatically associated with lymph node metastasis [OR: 2.61 (95CI: $0.99-6.88$ ); $P=0.05$ ] but high CD73 expression was not correlated with the other reported clinicopathological features including age, gender, smoking history, clinical stage and differentiation (Fig. 5).

\section{Sensitivity and publication bias}

Sensitivity analysis was conducted by deleting one study at one time to assess its effect on prevalence and pooled HRs. Deletion of the study by Martin et al. [21] and Yusuke et al. [26] slightly decreased the heterogeneity in the analysis of high CD73 expression prevalence. No other individual study influenced the results. Begg's funnel plots and Egger's tests evaluated the publication bias, and it was only detected in the analysis of high CD73 expression prevalence $(P<0.05$ for Egger's test). Further analyses showed that the Begg's funnel plot was symmetric and Egger's tests suggested that there was no evidence of publication bias (Additional file 1: Figure S20). 


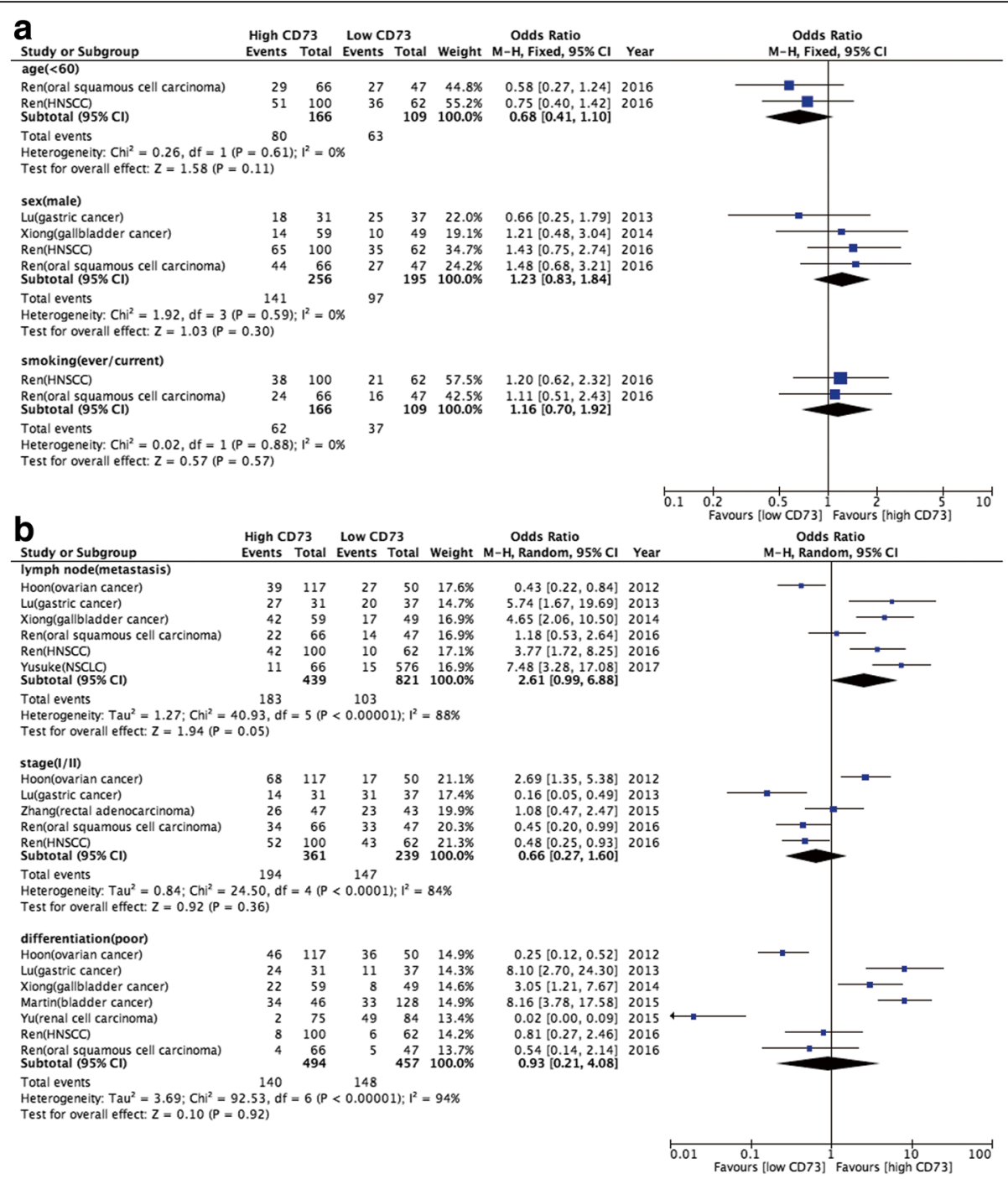

Fig. 5 The relationship between clinicopathological features and CD73 overexpression in different cancers. a meta-analysis of CD73 overexpression and age < 60 years-old, male gender and smoking history; (b) meta-analysis of CD73 overexpression and lymph node metastasis, clinical stage and tumor differentiation

\section{Discussion}

To our knowledge, the current study is the one of the first studies to comprehensively investigate the characterization of CD73 and its effect on prognosis in various solid tumors. In the current study, the pooled results showed that CD73 highly expressed in 12 types of human cancers and the prevalence of high CD73 expression was more than $50 \%$. Due to the limited number of included cases, we assessed the expression level of CD73 in a broader set of cancers versus matched normal tissues by using the web-based microarray database (Oncomine). The results further suggested that CD73 highly expressed in most kinds of cancers including bladder, brain, invasive lobular breast, esophageal, gastric, pancreatic cancer, rectal mucinous, renal cell, lung large cell, oral cavity squamous cell carcinoma, melanoma, and lung adenocarcinoma. However, several types of tumors (cervical, liver, colorectal, prostate invasive ductal breast, small cell lung cancer and lung squamous cell carcinoma) showed similar CD73 expression level compared to that in matched normal tissues. Notably, cecum adenocarcinoma or ovarian cancer had the lower CD73 expression level than that in matched normal tissue. Consistently, Lu et al. reported that high CD73 expression was found in $45.60 \%$ of patients with gastric cancer [11]. Yu and colleagues also found that $47.20 \%$ of renal cell carcinoma highly expressed CD73 [22]. Of note, Hoon et al. collected 167 patients with epithelial ovarian cancer and found that $70.1 \%$ of patients showed positive expression for CD73 while data from Oncomine showed that ovarian cancer had low CD73 expression level. The reason for this discrepancy may include that the 
histological type of included ovarian cancer and test methods are different. Histology of ovarian cancer in Oncomine were mucinous, serous, endometrioid and clear cell adenocarcinoma and CD73 expression were analyzed based on microarray analysis whereas Hoon' study enrolled epithelial ovarian carcinoma and used IHC to assess the expression of CD73. These results indicated that distinct histological types of cancers would have distinct CD73 expression.

Furthermore, we investigated the relationship between high CD73 expression and prognosis in different cancers. The results of all included studies demonstrated that high CD73 expression was significantly associated with poor OS but not RFS. In virtue of the high heterogeneity and small number of included studies, we performed the validation subgroup analysis via "The Kaplan-Meier plotter" (KM plotter) database which is capable to assess the effect of 54,675 genes on survival of patients with breast, lung, gastric and ovarian cancer [27]. The pooled results showed that high CD73 expression was markedly associated with poor OS in breast and ovarian cancer but favorable OS in lung and gastric cancer. In breast cancer, a previous study demonstrated that positive CD73 expression was correlated with longer DFS and OS, which was opposite to the results from KM plotter. Theoretically, cancer cells with high CD73 expression possessed higher aggressiveness and invasiveness [28]. Leth-Larsen and colleagues also showed that intense NT5E/CD73 IHC staining was more common for breast cancer patients with relapse and lymph node metastases [29]. Hence, it seems that high CD73 expression was likely to be associated with poor prognosis in breast cancer. In lung cancer, however, Yusuke et al. also reported the contrary results that high CD73 expression was an independent indicator of poor prognosis for OS and RFS. The reason underlying this discrepancy is unclear as there are few studies to deeply explore the functions of CD73 in immune cells and tumor microenvironment of lung cancer [3]. The complex signaling pathway downstream to CD73-adenosince in lung cancer cells should be investigated to provide a preciously mechanistic explanation [26]. Of note, data on CD73 expression from KM plotter was on the basis of gene expression data whereas the published articles utilized the IHC to assess CD73 expression level. As is known, gene expression level is not positively related to the corresponding protein expression level. Moreover, the cutoff value of positive CD73 expression is also different. These would result in the difference on the association between CD73 expression and prognosis.

As to the clinicopathological characteristics, we found that high CD73 expression was significantly associated with lymph node metastasis but not correlated with the other reported clinicopathological features including age, gender, smoking history, clinical stage and differentiation. Consistently, Lu et al. enrolled 68 patients with resected gastric carcinoma and found that overexpression of CD73 was positively associated with lymph node metastases $(P=0.003)$ [11]. Similar result in gallbladder cancer was reported by Xiong and colleagues [19]. Furthermore, Ren et al. collected 162 patients with head and neck squamous cell carcinoma (HNSCC) and highlighted that there was a direct relationship between CD73 expression and lymph node metastases $(P<$ 0.001). They further demonstrated that $\mathrm{CD} 73$ could promote HNSCC migration and invasion via adenosine A3R stimulation and the activation of EGF/EGFR signaling [24]. This could be one of the potential mechanism for the close relationship between CD73 expression and lymph node metastases.

Accumulating evidence indicates that CD73adenosine pathway plays a crucial role in cancer progression and immune escape. A series of studies suggested that CD73-derived adenosine could help to form immunosuppressive environment via dampening anti-tumor effect of immune cells, such as CD8+ positive $\mathrm{T}$ cells and NK cells [3, 30]. Stagg et al. firstly reported that targeted blockade of CD73 could reduce the tumor growth and metastasis in immunecompetent mice through the activation of adaptive anti-tumor immunity [31]. After that, emerging evidence highlights the critical role of CD73 in the regulation of MDSC expansion, M2 macrophages polarization and Treg inhibitory activity [4, 32-34]. Recently, several studies showed that CD73 expression on tumor cells weakened the immune response to PD-1/PD-L1 inhibitors [35, 36]. Allard et al. reported that anti-CD73 mono-antibody (mAb) dramatically enhanced the effect of anti-CTLA-4 and PD-1 inhibitors against colon, prostate and breast cancers in mice model [36]. Iannone and colleagues also found that blockade of CD73 could enhance efficacy of antiCTLA-4 in melanoma model [37]. Beavis et al. further showed that combination of CD73-A2A inhibition and anti-PD-1 mAb resulted in greater antitumor immune response through prolonged expression of IFN-gamma and granzyme B [35]. These results suggested that $\mathrm{CD} 73$ was a potential biomarker for response to anti-PD-1/PD-L1 treatment.

Targeting CD73 also showed the favorable antitumor effects in preclinical studies $[38,39]$. To date, several potent inhibitors or antibodies of CD73 have been discovered via high-throughput drug screenings. One of the most valuable drugs is MEDI9447. MEDI9447 could enhance the activity of PD-1 antibody in a syngeneic tumor model through increasing CD8+ $\mathrm{T}$ cells and reducing MDSC and Tregs in the tumor microenvironment [40, 41]. Herein, they performed a phase I clinical 
trial of the MEDI9447 in patients with advanced solid tumors, as single agent and in combination with the anti-PD-L1 antibody (NCT02503774). Another small molecular inhibitor, PBF-509, is the A2A receptor antagonist. A phase I study on PBF-509 in immunotherapy-naïve, locally advanced or metastatic NSCLC patients is ongoing (NCT02403193).

There are several limitations of our study. Firstly, the publication bias was inevitable. Several abstracts were identified but not further detailed in standard publications. Although we have tried our best to contact authors of primary studies, no reply was received. Therefore, we could not include these data. Secondly, the quality of data on the incidence of high CD73 expression was statistically heterogeneous among the studies. Thirdly, it is difficult to make a direct comparison between distinct studies due to several confounding factors including lab condition, test techniques and platform, definition of positive CD73 expression and so on.

\section{Conclusions}

In summary, the current study indicates that high CD73 expression would be a potential prognostic factor to human solid tumors, especially the lung, breast, gastric and ovarian cancer. High CD73 expression was correlated with distant/local lymph node metastases. CD73 is also a promising target in future cancer immunotherapy and has the potential significance as a biomarker for antiPD-1/PD-L1 treatment.

\section{Additional file}

Additional file 1: Figure S1. The expression level of CD73/NT5E in bladder cancer versus matched normal tissue. Figure S2. The expression level of CD73/NT5E in brain cancer versus matched normal tissue. Figure S3. The expression level of CD73/NT5E in breast cancer versus matched normal tissue. Figure S4. The expression level of CD73/NT5E in cervical cancer versus matched normal tissue. Figure S5. The expression level of CD73/NT5E in colorectal cancer versus matched normal tissue. Figure S6 The expression level of CD73/NT5E in esophageal cancer versus matched normal tissue. Figure S7. The expression level of CD73/NT5E in gastric cancer versus matched normal tissue. Figure $\mathbf{S 8}$. The expression level of CD73/NT5E in kidney cancer versus matched normal tissue. Figure S9. The expression level of CD73/NT5E in leukemia versus matched bone marrow. Figure S10. The expression level of CD73/NT5E in liver cancer versus matched normal tissue. Figure $\mathbf{S 1 1}$. The expression level of CD73/ NT5E in lung cancer versus matched normal tissue. Figure S12. The expression level of CD73/NT5E in lymphoma versus matched CD4+ T lymphocyte. Figure S13. The expression level of CD73/NT5E in melanoma versus matched normal tissue. Figure S14. The expression level of CD73/NT5E in myeloma versus matched plasma cell. Figure S15. The expression level of CD73/NT5E in oral cavity squamous cell carcinoma versus matched normal tissue. Figure S16. The expression level of CD73/NT5E in ovarian cancer versus matched normal tissue. Figure S17. The expression level of CD73/NT5E in pancreatic cancer versus matched normal tissue. Figure S18. The expression level of CD73/ NT5E in prostate cancer versus matched normal tissue. Figure S19. The expression level of CD73/NT5E in sarcoma versus matched normal tissue. Figure S20. Publication bias for the prevalence of CD73/NT5E in various cancers. (DOCX $721 \mathrm{~kb})$

\section{Abbreviations}

AMP: Adenosine monophosphate; Cl: Confidence interval; CTLA-4: Cytotoxic T-lymphocyte antigen-4; GPCR: G-protein coupled receptor; HNSCC: Head and neck squamous cell carcinoma; HR: high risk; IF: Immunofluorescence; IHC: Immunohistochemistry; mAb: Mono-antibody; NK: Natural killer; NSCLC: Non-small-cell lung cancer; NT5E: ecto-5'-nucleotidase; OS: Overall survival; PD-1: Programmed cell death protein-1; RFS: recurrence free survival

\section{Acknowledgements}

Not applicable.

\section{Funding}

The design of the study and collection, analysis, and interpretation of data and in writing the manuscript study was supported by grants from the National Natural Science Foundation of China (No. 81672286, 81372392 and 81402486), key project of Shanghai Municipal Commission of Health and Family Planning (No. 2013zyjb0401) and Outstanding Yong Doctor Program of Shanghai Municipal Commission of Health and Family Planning (No. XYQ2013097)

\section{Availability of data and materials}

The datasets used and/or analyzed during the current study are available from the corresponding author on reasonable request.

\section{Authors' contributions}

T.J., X.F.X. and C.C.Z. designed the study. X.F.X, M.Q., X.F.L., C.Z., F.Z., G.H.G., F.Y.W. and X.X.C. collected the relevant papers and data. T.J., X.F.X. and M.Q. performed the analysis. T.J., C.X.S., S.X.R., C.Y.Z. and C.C.Z. wrote the manuscript. C.Y.Z. and C.C.Z. revised the manuscript. All authors have read and approved the final version of this manuscript.

\section{Ethics approval and consent to participate}

None.

Consent for publication

Not Applicable.

\section{Competing interests}

The authors declare that they have no competing interests.

\section{Publisher's Note}

Springer Nature remains neutral with regard to jurisdictional claims in published maps and institutional affiliations.

\section{Author details}

${ }^{1}$ Department of Medical Oncology, Shanghai Pulmonary Hospital, Thoracic Cancer Institute, Tongji University School of Medicine, No. 507, Zheng Min Road, Shanghai 200433, People's Republic of China. ${ }^{2}$ Department of Clinical Laboratory, Fudan University Shanghai Cancer Center, Shanghai 200032, People's Republic of China. ${ }^{3}$ Department of Oncology, Shanghai Medical College, Fudan University, Shanghai 200032, People's Republic of China. ${ }^{4}$ Department of Lung Cancer and Immunology, Shanghai Pulmonary Hospital, Thoracic Cancer Institute, Tongji University School of Medicine, Shanghai 200433, People's Republic of China. ${ }^{5}$ Department of Medical Oncology, Yancheng TCM Hospital Affiliated to Nanjing University of Chinese Medicine, Yancheng 224001, People's Republic of China.

Received: 21 February 2017 Accepted: 29 January 2018

Published online: 07 March 2018

\section{References}

1. Allard D, Turcotte M, Stagg J. Targeting A2 adenosine receptors in cancer. Immunol Cell Biol. 2017:95(4):333-9.

2. Antonioli L, Pacher P, Vizi ES, Hasko G. CD39 and CD73 in immunity and inflammation. Trends Mol Med. 2013;19:355-67.

3. Allard D, Allard B, Gaudreau PO, et al. CD73-adenosine: a next-generation target in immuno-oncology. Immunotherapy. 2016;8:145-63.

4. Beavis PA, Stagg J, Darcy PK, Smyth MJ. CD73: a potent suppressor of antitumor immune responses. Trends Immunol. 2012;33:231-7.

5. Stagg J, Smyth MJ. Extracellular adenosine triphosphate and adenosine in cancer. Oncogene. 2010;29:5346-58. 
6. Allard B, Turcotte M, Spring $\mathrm{K}$, et al. Anti-CD73 therapy impairs tumor angiogenesis. Int J Cancer. 2014;134:1466-73.

7. Gao ZW, Wang HP, Lin F, et al. CD73 promotes proliferation and migration of human cervical cancer cells independent of its enzyme activity. BMC Cancer. 2017;17:135.

8. Blay J, White TD, Hoskin DW. The extracellular fluid of solid carcinomas contains immunosuppressive concentrations of adenosine. Cancer Res. 1997;57:2602-5.

9. Antonioli L, Yegutkin GG, Pacher P, et al. Anti-CD73 in cancer immunotherapy: awakening new opportunities. Trends Cancer. 2016;2:95-109.

10. Supernat A, Markiewicz A, Welnicka-Jaskiewicz M, et al. CD73 expression as a potential marker of good prognosis in breast carcinoma. Appl Immunohistochem Mol Morphol. 2012;20:103-7.

11. Lu XX, Chen YT, Feng B, et al. Expression and clinical significance of CD73 and hypoxia-inducible factor-1alpha in gastric carcinoma. World J Gastroenterol. 2013;19:1912-8.

12. Zhang $B$, Song $B$, Wang $X$, et al. The expression and clinical significance of CD73 molecule in human rectal adenocarcinoma. Tumour Biol. 2015;36: 5459-66.

13. Liberati A, Altman DG, Tetzlaff J, et al. The PRISMA statement for reporting systematic reviews and meta-analyses of studies that evaluate health care interventions: explanation and elaboration. Ann Intern Med. 2009;151:W65-94.

14. Jiang $T$, Wang $Y$, Zhou $F$, et al. Prognostic value of high EZH2 expression in patients with different types of cancer: a systematic review with metaanalysis. Oncotarget. 2016;7:4584-97.

15. Tierney JF, Stewart LA, Ghersi D, et al. Practical methods for incorporating summary time-to-event data into meta-analysis. Trials. 2007:8:16

16. Oh HK, Sin Jl, Choi J, et al. Overexpression of CD73 in epithelial ovarian carcinoma is associated with better prognosis, lower stage, better differentiation and lower regulatory T cell infiltration. J Gynecol Oncol. 2012; 23:274-81.

17. Wu XR, He XS, Chen YF, et al. High expression of CD73 as a poor prognostic biomarker in human colorectal cancer. J Surg Oncol. 2012;106:130-7.

18. Loi S, Pommey S, Haibe-Kains B, et al. CD73 promotes anthracycline resistance and poor prognosis in triple negative breast cancer. Proc Natl Acad Sci U S A. 2013;110:11091-6.

19. Xiong L, Wen Y, Miao X, Yang Z. NT5E and FcGBP as key regulators of TGF1-induced epithelial-mesenchymal transition (EMT) are associated with tumor progression and survival of patients with gallbladder cancer. Cell Tissue Res. 2014;355:365-74.

20. Turcotte M, Spring K, Pommey S, et al. CD73 is associated with poor prognosis in high-grade serous ovarian cancer. Cancer Res. 2015;75:4494-503.

21. Wettstein MS, Buser L, Hermanns T, et al. CD73 predicts favorable prognosis in patients with nonmuscle-invasive urothelial bladder cancer. Dis Markers. 2015;2015:785461.

22. $\mathrm{Yu}$ Yl, Wang $W$, Song $\mathrm{L}$, et al. Ecto-5'-nucleotidase expression is associated with the progression of renal cell carcinoma. Oncol Lett. 2015;9:2485-94.

23. Leclerc BG, Charlebois R, Chouinard G, et al. CD73 expression is an independent prognostic factor in prostate cancer. Clin Cancer Res. 2016;22: 158-66.

24. Ren ZH, Lin CZ, Cao W, et al. CD73 is associated with poor prognosis in HNSCC. Oncotarget. 2016;7:61690-702.

25. Ren ZH, Yuan YX, Ji T, Zhang CP. CD73 as a novel marker for poor prognosis of oral squamous cell carcinoma. Oncol Lett. 2016;12:556-62.

26. Inoue $\mathrm{Y}$, Yoshimura $\mathrm{K}$, Kurabe $\mathrm{N}$, et al. Prognostic impact of CD73 and A2A adenosine receptor expression in non-small-cell lung cancer. Oncotarget. 2017;8:8738-51.

27. Szasz AM, Lanczky A, Nagy A, et al. Cross-validation of survival associated biomarkers in gastric cancer using transcriptomic data of 1,065 patients. Oncotarget. 2016;7:49322-33.

28. Zhou X, Zhi X, Zhou P, et al. Effects of ecto-5'-nucleotidase on human breast cancer cell growth in vitro and in vivo. Oncol Rep. 2007;17:1341-6.

29. Leth-Larsen R, Lund R, Hansen HV, et al. Metastasis-related plasma membrane proteins of human breast cancer cells identified by comparative quantitative mass spectrometry. Mol Cell Proteomics. 2009; 8:1436-49.

30. Cekic C, Linden J. Adenosine A2A receptors intrinsically regulate CD8+ T cells in the tumor microenvironment. Cancer Res. 2014;74:7239-49.

31. Stagg J, Divisekera U, McLaughlin N, et al. Anti-CD73 antibody therapy inhibits breast tumor growth and metastasis. Proc Natl Acad Sci U S A. 2010;107:1547-52.
32. Ryzhov S, Novitskiy SV, Goldstein AE, et al. Adenosinergic regulation of the expansion and immunosuppressive activity of CD11b+Gr1+ cells. J Immunol. 2011;187:6120-9.

33. Ferrante CJ, Pinhal-Enfield G, Elson G, et al. The adenosine-dependent angiogenic switch of macrophages to an M2-like phenotype is independent of interleukin-4 receptor alpha (IL-4Ralpha) signaling. Inflammation. 2013;36: 921-31.

34. Li MQ, Wang Y, Chang KK, et al. CD4+Foxp3+ regulatory T cell differentiation mediated by endometrial stromal cell-derived TECK promotes the growth and invasion of endometriotic lesions. Cell Death Dis. 2014;5:e1436.

35. Beavis PA, Milenkovski N, Henderson MA, et al. Adenosine receptor $2 A$ blockade increases the efficacy of anti-PD-1 through enhanced antitumor Tcell responses. Cancer Immunol Res. 2015;3:506-17.

36. Allard B, Pommey S, Smyth MJ, Stagg J. Targeting CD73 enhances the antitumor activity of anti-PD-1 and anti-CTLA-4 mAbs. Clin Cancer Res. 2013; 19:5626-35.

37. lannone $\mathrm{R}$, Miele $\mathrm{L}$, Maiolino $\mathrm{P}$, et al. Adenosine limits the therapeutic effectiveness of anti-CTLA4 mAb in a mouse melanoma model. Am J Cancer Res. 2014:4:172-81.

38. Iqbal J, Saeed A, Raza R, et al. Identification of sulfonic acids as efficient ecto-5'-nucleotidase inhibitors. Eur J Med Chem. 2013;70:685-91.

39. Bhattarai S, Freundlieb M, Pippel J, et al. Alpha,beta-methylene-ADP (AOPCP) derivatives and analogues: development of potent and selective ecto-5'-Nucleotidase (CD73) inhibitors. J Med Chem. 2015;58:6248-63.

40. Hay C SE, Huang Q et al. MEDI9447: enhancing anti-tumor immunity by targeting CD73 in the tumor microenviroment. Presented at: 106th annual meeting of the American Association for Cancer Research. Philadelphia, PA, USA, 18-22 April 2015.

41. Hay CM, Sult E, Huang Q, et al. Targeting CD73 in the tumor microenvironment with MEDI9447. Oncoimmunology. 2016;5:e1208875.

\section{Submit your next manuscript to BioMed Central and we will help you at every step:}

- We accept pre-submission inquiries

- Our selector tool helps you to find the most relevant journal

- We provide round the clock customer support

- Convenient online submission

- Thorough peer review

- Inclusion in PubMed and all major indexing services

- Maximum visibility for your research

Submit your manuscript at www.biomedcentral.com/submit

Biomed Central 